\title{
EL ENFOQUE INTERDISCIPLINARIO: UN RETO PARA LA DIDÁCTICA DE LA MATEMÁTICA EN CUBA
}

\author{
Raidy Teidy Rojas Angel Bello ${ }^{1}$ \\ Aneya llizastigui Matos ${ }^{2}$ \\ Carmen Alvarado Romero ${ }^{3}$
}

\begin{abstract}
Resumen
Constituye un reto para la didáctica de la Matemática en la carrera Matemática-Física, formar un profesional revolucionario con una preparación político-ideológica y científico-metodológica que le permita dirigir el proceso educativo y, en particular, el proceso de enseñanza-aprendizaje de la Matemática y la Física con un enfoque interdisciplinario. El presente artículo tiene como objetivo describir un proceder metodológico para lograr el enfoque interdisciplinario desde las potencialidades del tema de Didáctica de la Matemática: tratamiento de ejercicios y problemas en función de la dirección eficiente del proceso de enseñanza-aprendizaje de la Matemática la educación media básica.
\end{abstract}

Palabras-claves: Enfoque interdisciplinario. Ejercicios y problemas matemáticos. Proceder metodológico.

\section{ABORDAGEM INTERDISCIPLINAR: UM DESAFIO PARA O ENSINO DE MATEMÁTICA EM CUBA}

\section{Resumo}

Um desafio para o ensino de matemática na carreira Matemática-Física é formar um profissional revolucionário, com uma preparação político-ideológica, científica e metodológica que permita direcionar o processo educacional e, em especial, o processo de ensino e aprendizagem da matemática Física em uma abordagem interdisciplinar. Este artigo tem como objetivo descrever uma abordagem metodológica para alcançar um enfoque interdisciplinar a partir do potencial do tópico Ensino da Matemática: tratamento de exercícios e problemas, com base na gestão eficiente do ensino e aprendizagem de matemática do ensino secundário básico.

\footnotetext{
${ }^{1}$ Profesora asistente de la Universidad de Ciencias Pedagógicas "Manuel Ascunce Domenech", en la provincia de Ciego de Ávila, Cuba. Es Licenciada en Educación, Especialidad Matemática-Computación; Máster en Ciencias de la Educación Superior y Doctora en Ciencias Pedagógicas (2013). Jefa del departamento de Matemática- Física y Miembro del Centro de Estudio de Evaluación de la Calidad. Ha publicado artículos en revistas nacionales e internacionales y es investigadora de los proyectos de investigación de Pedagogía y El perfeccionamiento del proceso de enseñanza-aprendizaje de la Matemática y la Física. E-mail: <raidyra@ucp.rimed.cu>.

2 Profesora Titular y Doctora en Ciencias Pedagógicas, Licenciada en Educación Especialidad MatemáticaComputación, actualmente se desempeña como jefa de carrera de Matemática-Física de la Facultad de Ciencias de la Universidad de Ciencias Pedagógicas “Manuel Ascunce Domenech" de la provincia de Ciego de Ávila. . Ha publicado artículos en revistas nacionales e internacionales y es investigadora de los proyectos de investigación de Pedagogía y El perfeccionamiento del proceso de enseñanza-aprendizaje de la Matemática y la Física. E-mail: <aneya76@gmail.com>.

3 Profesora auxiliar y Máster en Ciencias de la Educación Superior, Licenciada en Educación Especialidad Matemática, actualmente se desempeña como jefa de disciplina de Análisis Matemático de la carrera de Matemática-Física de la Facultad de Ciencias de la Universidad de Ciencias Pedagógicas "Manuel Ascunce Domenech" de la provincia de Ciego de Ávila. Ha publicado artículos en revistas nacionales e internacionales y es investigadora del proyecto El perfeccionamiento del proceso de enseñanza-aprendizaje de la Matemática y la Física. E-mail: <carmenar@ucp.ca.rimed.cu>.
} 
Palavras-chaves: Abordagem interdisciplinar. Problemas e exercícios matemáticos. Procedimento metodológico.

\title{
INTERDISCIPLINARY APPROACH: A CHALLENGE FOR MATHEMATICS TEACHING IN CUBA
}

\begin{abstract}
A challenge for Mathematics teaching in Mathematics-Physics carrier is train a revolutionary professional, with politicalideological, scientific and methodological preparation to allow orientate all the education process, specially Mathematics and Physics teaching and learning process in an interdisciplinary approach. Thi $\mathrm{s}$ issue had as aim to describe a methodological approach to achieve an interdisciplinary approach fro $\mathrm{m}$ the potential theme Mathematics teaching: deal with exercises and problems based on the efficien $\mathrm{t}$ manage of Mathematics teaching and learning in the High School.
\end{abstract}

Keywords: Interdisciplinary approach; Mathematics Problems and exercises; MethodologicalProcedu re.

\section{INTRODUCCIÓN:}

La Didáctica de la Matemática es la encargada de perfeccionar la dirección del proceso de enseñanza-aprendizaje de esta ciencia, de manera que se corresponda con las exigencias establecidas para los programas de esta asignatura para cada Educación. Al analizar el Modelo del profesional del Licenciado en Educación especialidad Matemática-Física podemos constatar

[...] se pretende formar un profesional revolucionario con una preparación político-ideológica y científico-metodológica que le permita dirigir el proceso educativo y en particular, el proceso de enseñanza-aprendizaje de la Matemática y la Física con un enfoque interdisciplinario pasado en las relaciones con la ciencia, la tecnología, la sociedad y el medio ambiente, en las condiciones de la Revolución Cubana (COLECTIVO DE AUTORES, 2010, P. 8).

Al indagar en las investigaciones desarrolladas en Cuba en los últimos años, acerca de las reflexiones teórico-metodológicas para el proceso de enseñanza-aprendizaje de la Matemática con enfoque interdisciplinario, se consideran los nodos cognitivos como un elemento esencial para lograr la interdisciplinariedad (HERNÁNDEZ, 1989; 1995; ÁLVAREZ, 1999, 2002). 
En las investigaciones pedagógicas consultadas (ÁLVAREZ, 1999; PERERA, 2000; CABALLERO, 2000; FERNÁNDEZ, DE ALIAZA, 2000), por lo general predomina el estudio de la interdisciplinariedad en el proceso de enseñanza-aprendizaje fundamentado en la formación integral del estudiante, y desde la concepción del profesor por asignatura, pero no existen referentes sobre el análisis vinculado a la formación del Licenciado en Matemática-Física para el tratamiento interdisciplinario del contenido matemático.

El análisis de investigaciones referidas al tema (HERNÁNDEZ, 1989, 1995; ÁLVAREZ, 1999, 2002), los resultados de observaciones a clases y a actividades metodológicas, la consulta bibliográfica de las principales obras de la Didáctica de la Matemática y la experiencia de las autoras como profesora del colectivo de Didáctica de la Matemática permitieron identificar insuficiencias en la práctica pedagógica y carencias teóricas, que limitan la dirección del proceso de enseñanza-aprendizaje de la Matemática con un enfoque interdisciplinario:

- Insuficiencias en la práctica pedagógica:

Es limitado el trabajo con las líneas directrices de la enseñanza de la Matemática en la disciplina.

Es deficiente el tratamiento de los ejes transversales y programas directores de la escuela desde las clases de Didáctica de Matemática.

D En el proceso de enseñanza-aprendizaje de la disciplina no se enseña la determinación de los nexos interdisciplinarios de la Matemática Escolar con otras asignaturas del grado.

- Carencias teóricas en la Didáctica de la Matemática se manifiestan:

El enfoque disciplinar en el tratamiento de los componentes del proceso de enseñanza-aprendizaje de la Matemática (nivel general), en el tratamiento de situaciones típicas de la enseñanza de la Matemática (nivel particular) y en el tratamiento de complejos de materias (nivel singular). En el primer nivel los procedimientos existentes para determinar las relaciones entre los contenidos en la asignatura Matemática (cortes verticales y cortes horizontales a los programas) (BALLESTER, 1992) tienen un alcance solo disciplinar, es decir, no más allá de las fronteras de la Matemática. Ello provoca que la disciplina esté centrada básicamente en el análisis de los contenidos y métodos de la asignatura, sin atender con la sistematicidad necesaria los niveles de integración, que deben tener con los contenidos de las 
demás asignaturas del currículo con los contenidos de los programas directores y con los contenidos principales para lograr los objetivos formativos.

Teniendo en cuenta lo anteriormente expresado, se declara como problemática: Insuficiencia en el proceder metodológico de los docentes de la disciplina Didáctica de la Matemática para lograr el enfoque interdisciplinario en el tratamiento del contenido, en función de las exigencias del modelo del profesional de la carrera Matemática-Física. La causa fundamental es la deficiente preparación metodológica de los docentes del colectivo de disciplina de Didáctica de la Matemática para lograr el enfoque interdisciplinario en el tratamiento del contenido de la educación media. Para esto se propone como objetivo del artículo: describir un proceder metodológico para lograr el enfoque interdisciplinario desde las potencialidades del tema de Didáctica de la Matemática: tratamiento de ejercicios y problemas, en función de la dirección eficiente del proceso de enseñanza-aprendizaje de la Matemática la educación media básica.

\section{DESARROLLO}

El Dr. C. Jorge Fiallo reveló en su obra que las relaciones interdisciplinarias constituyen una vía efectiva para contribuir al logro de la relación mutua del sistema de hechos, fenómenos, conceptos, leyes y teorías que se abordan en la escuela y permiten garantizar un sistema general de conocimientos y habilidades, tanto de carácter intelectual como práctico, un sistema de valores, convicciones y relaciones hacia el mundo real y objetivo, lo que permite desarrollar en los estudiantes una cultura general con la finalidad de prepararse integralmente para la vida social (FIALLO, 2001).

Este autor refiere que la interdisciplinariedad es uno de los niveles para lograr las relaciones interdisciplinarias y la conceptualiza como:

la cooperación entre varias disciplinas e interacciones que provocan enriquecimientos mutuos. Estas interacciones pueden ir desde la simple comunicación de ideas hasta la integración mutua de leyes, teorías, hechos, conceptos, habilidades, hábitos, normas de conductas, sentimientos, valores a desarrollar, metodologías, formas de organización de las actividades e inclusive de organización de las investigaciones (FIALLO, 2001, p. 51). 
Otros investigadores han abordado la interdisciplinariedad en el proceso de enseñanza-aprendizaje, haciendo énfasis en la necesidad de vincular el aprendizaje con situaciones vivenciales de los estudiantes, para la comprensión lógica del contenido y posibilitar, así, la estructuración de los conocimientos en los planes de estudio y diseños curriculares (TORRES, 1994; FIALLO, 1996; MAÑALICH, 1998; NÚÑEZ, 1999; PERERA, 2000; CABALLERO, 2000; ÁLVAREZ, 1999, 2003; GUTIÉRREZ, 2002).

Se destacan otras investigaciones que no solo han asumido la interdisciplinariedad en el proceso de enseñanza-aprendizaje, sino que han propiciado desarrollar la misma desde el trabajo metodológico, y que son referentes teóricos de la presente investigación (FIALLO, 2001):

- La utilización de los ejes transversales en el proceso de enseñanza-aprendizaje, introducida en el curso 1995-1996, encaminados a la Educación Patriótica, Militar e Internacionalista, Educación Laboral y por la Eficiencia Económica, Educación Ambiental y de Ahorro de Energía, Educación para la Salud y por una Sexualidad responsable, los que desempeñan un papel fundamental como contenidos culturales relevantes, necesarios para la vida y la convivencia, en la formación integral que demanda cada sociedad a través de una educación en valores, para que los estudiantes se sensibilicen y asuman posiciones correctas ante los problemas y sean capaces de enjuiciarlos de manera crítica.

* La aplicación de los programas directores facilita otra de las formas de lograr la interdisciplinariedad, considerando los principales contenidos de las disciplinas Matemática, Lengua Materna e Historia constituyendo documentos rectores que guían la planificación, ejecución y evaluación de las acciones de las disciplinas que se imparten en esta educación, de manera que se alcancen los objetivos propuestos. Se implementan en el proceso de enseñanza-aprendizaje de las diferentes asignaturas del currículum en el curso escolar 19992000, para el nivel de Secundaria Básica. Los programas directores establecen, por grados, aspectos comunes que son de obligatorio cumplimiento para cada una de las disciplinas que conforman el plan de estudio, con la finalidad de formar un estudiante preparado para entender su contexto con los conocimientos necesarios de cada ciencia.

* Los contenidos comunes de las disciplinas también han propiciado la interdisciplinariedad desde el trabajo metodológico. Diferentes investigadores han hecho alusión a este tema, entre ellos se puede citar a García (2001): precisa el interobjeto como un 
ente común de las asignaturas, lo considera objeto del trabajo interdisciplinario, lo ubica en el sistema de conocimientos, habilidades, valores y métodos en la Educación Técnica y Profesional; Salazar, (2001) define el interobjeto como el elemento esencial asumido por las disciplinas que integran el currículo de una carrera universitaria.

La Dra. Marta Güemez, a diferencia de García y Salazar; considera los interobjetos como lo común esencial entre los contenidos de la Secundaria Básica en estrecha relación con las habilidades, valores y actitudes en la formación inicial del Profesor General Integral, de modo que ellos puedan contribuir a la solución de problemas profesionales; esta propuesta está dirigida a la preparación interdisciplinaria de los profesores de la facultad de Profesor General Integral (GÜEMEZ, 2005).

En las investigaciones referidas anteriormente y en otras sobre este tema (SAGÓGUIBO, 2002; GÓMEZ, 2006) se destaca la necesidad de la interdisciplinariedad, desde el trabajo metodológico en los departamentos y consejo de grado, con un carácter planificado y cooperado del colectivo pedagógico, y como vía común de preparación para desarrollar un proceso de enseñanza-aprendizaje con enfoque interdisciplinario.

La determinación de líneas directrices constituye otra de las vías para potenciar la interdisciplinariedad desde la preparación metodológica (FIALLO, 2001). En el análisis de la interdisciplinariedad es necesario considerar el componente contenido del proceso de enseñanza-aprendizaje con un enfoque dialéctico de los fenómenos, hechos y procesos estudiados para posibilitar que las distintas asignaturas aborden diferentes aristas de problemas comunes sobre la realidad natural y social.

El contenido como componente del proceso de enseñanza-aprendizaje es un aspecto esencial para profundizar en la interdisciplinariedad, apreciándose en los últimos años como tendencia un nivel de integración de sus dimensiones: conocimientos, habilidades, valores, actitudes y la necesidad de transmitirlo desde un enfoque interdisciplinario (DANILOV, 1975; KLINGBERG, 1980; LABARRERE, 1987; CASTRO, 1999; ÁLVAREZ DE Z, 1999; ÁlVAREZ, 1995, 1997; ADDINE,1998, 2004; PLA, 2000; HERNÁNDEZ, 2000; SILVESTRE Y ZILBERSTEIN, 2002).

Los contenidos de la enseñanza de la Matemática son aquellos conocimientos (conceptos, teoremas y procedimientos), habilidades, hábitos, capacidades, experiencia transformadora (investigativa), valores, convicciones y normas de conductas que al ser 
asimiladas por los estudiantes, en el aprendizaje, posibilitan el cumplimiento de los objetivos planteados.

El contenido se subordina a los objetivos, pero estos se manifiestan, se concretan con ayuda del contenido. Esta relación dialéctica entre el contenido (como fenómeno) y el objetivo (como esencia) tiene un significativo valor metodológico para la dirección del PEAM.

A partir de la determinación de los objetivos formativos por grado para la Secundaria Básica, se precisa la necesidad de vincular los contenidos matemáticos con la vida y la responsabilidad que tiene en el desarrollo del pensamiento lógico para contribuir a la formación integral del estudiante, lo que exige el enfoque interdisciplinario del proceso de enseñanza-aprendizaje de la Matemática.

Una de las formas para potenciar la interdisciplinariedad en el proceso de enseñanza aprendizaje es a partir de las Matemáticas (FIALLO, 2001). La interdisciplinariedad en el proceso de enseñanza-aprendizaje de la Matemática facilita profundizar en la cientificidad del contenido y la relación de la teoría con la práctica, permite al estudiante transitar por los diferentes niveles de asimilación; aplicar los conocimientos y habilidades matemáticas en su modo de actuación; así como solucionar problemas de su contexto.

Las investigaciones sobre la interdisciplinariedad en el proceso de enseñanzaaprendizaje de la Matemática coinciden en la determinación de los denominados nodos como una de las formas particulares o específicas para lograrla (HERNÁNDEZ, 1989; ÁLVAREZ, 1999; FERNÁNDEZ DE ALAIZA, 2000).

Para las autoras estas definiciones no rebasan el marco de los conocimientos matemáticos, y se plantea una nueva concepción definida por la Dra. C Ilizastigui (2009), en su tesis doctoral, donde se definen

los nexos interdisciplinarios son el reflejo consecuente de las relaciones objetivas existentes entre los objetos, fenómenos y procesos de la naturaleza, la sociedad y el pensamiento, en el contenido de las diferentes disciplinas. [...] El nodo interdisciplinario es un contenido en torno al cual converge al menos un contenido de otra asignatura del currículo para sistematizar los nexos interdisciplinarios en el proceso de enseñanza aprendizaje [y] El enfoque interdisciplinario es asumir la sistematización de los nexos en el proceso de enseñanza aprendizaje a partir de los nodos interdisciplinarios identificado" . 
Para la determinación de estos nodos interdisciplinarios del contenido matemático se debe tener en cuenta los siguientes pasos:

- Estudiar el currículo y programas de asignaturas del grado y sus potencialidades para establecer los nexos interdisciplinarios a partir de los contenidos que se reflejan desde el punto de vista cronológico.

- Estudiar los documentos rectores (programas directores, ejes transversales y líneas directrices de la Matemática), específicamente los objetivos y contenidos que se proponen.

- Determinar los contenidos principales en torno al cual convergen contenidos de las otras asignaturas, programas directores y ejes transversales a partir del trabajo cooperado entre los profesores del colectivo de grado.

Los ejercicios y problemas son unas de las líneas directrices que atraviesan todas las disciplinas de Matemática de la formación de este profesional y a la vez es una situación típica estudiada por la Didáctica de la Matemática. Para lograr el enfoque interdisciplinario en el tratamiento de este contenido, el profesor de Didáctica debe proceder metodológicamente atendiendo a los siguientes pasos:

1. Concebir actividades laborales e investigativas que le permita a los estudiantes sistematizar las relaciones interdisciplinarias, a partir de :

a. Determinar la relación del contenido matemático con los ejes transversales, los programas directores y las líneas directrices, según el objetivo de la unidad y las características de los estudiantes.

b. Determinar los nexos interdisciplinarios, a partir de los nodos mediante la aplicación de un corte horizontal ampliado a los contenidos del tema.

2. Concebir tareas docentes que le permita al estudiante seleccionar, formular y tratar metodológicamente ejercicios y problemas con enfoque interdisciplinario de la Matemática Escolar, teniendo en cuenta:

a. Las tareas integradoras.

b. Las tareas docentes interdisciplinarias. 
3. Implicar a los estudiantes en reflexiones metacognitivas del proceder utilizado como sustento de su futuro desempeño profesional.

Ejemplos de tareas docentes interdisciplinarias en el tratamiento de los ejercicios y problemas de la Didáctica de la Matemática, utilizados son:

Del siguiente problema matemático:

En una vivienda tenían 6 bombillos incandescentes y 2 bombillos ahorradores, por lo que se consumía diariamente $400 \mathrm{~W}$. Al sustituir 4 de los bombillos incandescentes por bombillos ahorradores el consumo de electricidad se redujo $160 \mathrm{~W}$ menos. ¿Cuántos watt consume cada tipo de esos bombillos?

- Explica la importancia del ahorro de electricidad y de combustible.

- Explicar por qué la explotación irracional del petróleo causa daños al medio ambiente.

- Investiga la cantidad de petróleo que se consume para generar un KW y cuál es el precio de cada barril en el mercado internacional.

El profesor orienta al estudiante sobre las siguientes acciones:

1. Precise el contenido matemático.

2. Identifique que nexos interdisciplinarios se sistematizan en el problema.

3. Precise cuales ejes transversales se le da salida en el problema.

4. Realice el tratamiento metodológico, a partir de lo estudiado en el programa heurístico general.

5. Intercambia con tus compañeros el tratamiento metodológico realizado.

Otra tarea docente utilizada es:

Seleccione del libro de texto y cuaderno complementario de octavo grado, tres problemas matemáticos para una clase de ejercitación de la temática 3.2 de noveno grado (Procedimientos analíticos para resolver sistemas de dos ecuaciones lineales con dos variables. Problemas), donde se potencie el enfoque interdisciplinario.

1.1. Reflexiona con tus compañeros sobre el proceder para lograr el enfoque interdisciplinario en el tratamiento del contenido matemático

Impulsos: tener en cuenta

- Los nexos interdisciplinarios establecidos en el grado. 
- Los ejes transversales y programas directores.

- El criterio de selección estudiado.

- Los niveles de desempeño cognitivo.

Como actividad para su práctica laboral:

Indague con profesores de Matemática de la práctica laboral, cómo realizan la selección de ejercicios y problemas para su tratamiento en las clases y cuáles son las fuentes que más utilizan

Formule un problema matemático de la vida práctica para el tema "Ecuaciones lineales y problemas", de octavo grado.

- Indagar con los profesores de Matemática de su práctica laboral los nexos interdisciplinarios determinados en el trabajo metodológico del grado y realice él siguiente procedimiento para su formulación.

- Precise el contenido matemático y sus nexos interdisciplinarios sobre lo que vas a formular el problema. Recuerde el nivel de desempeño para el cual formula, la salida de uno o más ejes transversales vinculados con la vida práctica.

- Completa los elementos estructurales de un problema matemático a partir de datos reales de la vida práctica, teniendo en cuenta si es necesario realizar un gráfico o esquema, elaborar una o varias preguntas.

- Precise y redacta el problema atendiendo al contenido matemático y sus nexos interdisciplinarios, con claridad, coherencia y lógica. En el que tenga cuidado de la limpieza, orden, ortografía y redacción.

- Resuelve, comprueba y evalúa el problema elaborado.

- Intercambia con tus compañeros el problema formulado atendiendo a los requisitos estudiados.

- Planifique una clase donde se resuelva el problema formulado.

\section{CONCLUSIONES:}


- El enfoque interdisciplinario en la dirección del PEAM es una exigencia del modelo del profesional y de los programas de la escuela para la formación del licenciado en Educación especialidad Matemática-Física.

- El tema tratamiento de ejercicios y problemas tiene potencialidades para lograr el enfoque interdisciplinario.

- El proceder metodológico para lograr el enfoque interdisciplinario en el tratamiento del contenido concretado en el tema ejercicios y problemas es generalizable al resto de los temas de la disciplina.

\section{REFERENCIAS}

ADDINE, Fátima: Un modelo para las relaciones interdisciplinarias en la formación del profesional de perfil amplio. Proyecto. Impresión ligera. ISP E. J. Varona. (2000)

ADDINE, Fátima: La interacción: Núcleo de las Relaciones interdisciplinarias en la formación de profesores de perfil amplio. [Documento Digitalizado]. (2003)

ÁLVAREZ DE ZAYAS, CARLOS M. La Escuela en la vida. La Habana: Ed Pueblo y Educación. (2004)

ÁLVAREZ GÓMEZ, Aida. La realización de la relación intermateria de la Matemática y la Física en el proceso de enseñanza de la Matemática en la escuela cubana. Tesis de Doctorado. Minsk, Universidad Estatal. (1984)

ÁLVAREZ PÉREZ, MARTHA. Sí a la interdisciplinariedad. / M. Álvarez. --- En: Educación -- No. 97 - Cuba. (1999)

ÁLVAREZ PÉREZ, MARTHA Interdisciplinariedad: Una aproximación desde la EnseñanzaAprendizaje de las Ciencias /. Martha Álvarez Pérez. -La Habana: Ed Pueblo y Educación. (2003)

BALLESTER PEDROSO, SERGIO. Metodología de la enseñanza de la Matemática /. Sergio Ballester Pedroso, Hilario Santana de Armas. -La Habana: Ed Pueblo y Educación. (1992)

CABALLERO CAMEJO, Cayetano Alberto. La interdisciplinariedad de la Biología y la Geografía con la Química: Una estructura didáctica. Tesis de Doctorado. La Habana. (2000)

COLECTIVO DE AUTORES. Modelo del profesional plan de estudio "D", Carrera de Licenciatura en Educación Matemática-Física. La Habana, Cuba: Ministerio de Educación; 2010. p.8. 
DAVIDSON, L. J. Y REGUERA, R. “iQue todos los maestros cubanos sean como estos!” En: Revista Educación No.86. Editorial Pueblo y Educación. Ciudad de la Habana. (1995)

FERNÁNDEZ, DE ALAIZA. B Las interdisciplinariedad como base de una estrategia para el perfeccionamiento del diseño curricular de una carrera de ciencias técnicas y su aplicación a la Ingeniería en Automática en la República de Cuba. Tesis en opción al grado científico de Doctor en Ciencias Pedagógicas. Ciudad de la Habana. (2000)

FIALLO RODRÍGUEZ, J. La relación intermaterias: Una vía para incrementar la calidad de la Educación. Editorial Pueblo y Educación. La Habana. (1996)

FIALLO RODRÍGUEZ, J. La interdisciplinariedad en la escuela: de la utopía a la realidad. Curso 01/ Jorge Fiallo Rodríguez, Ciudad de la Habana (2001)

GUTIÉRREZ MORENO, R. Precisiones metodológicas para el trabajo con los objetivos formativos. "Félix Varela Morales", Villa Clara, 2004. (En soporte magnético) (2002)

HERNÁNDEZ FERNÁNDEZ, HERMINIA. El perfeccionamiento de la enseñanza de la Matemática Superior Cubana. Experiencias en el Álgebra Lineal. Tesis de Doctor en ciencias Pedagógicas. MES, Ciudad de la Habana. (1989)

ILIZASTIGUI MATOS, ANEYA. La preparación metodológica del PGI en el tratamiento del contenido matemática. Tesis en opción al grado científico de Doctor en Ciencias Pedagógicas. Villa Clara. (2009)

ILIZASTIGUI MATOS, ANEYA. Proceder metodológico para la dirección del proceso de enseñanza-aprendizaje de la Matemática con enfoque interdisciplinario en la Secundaria Básica. Editado en Revista IPLAC, Publicación Latinoamericana y Caribeña de Educación, con dirección www.revista.iplac.rimed.cu, con RNPS No. 2140/ ISSN 1993-6850, correspondiente a No. 2 Marzo-Abril 2013 sección artículos científicos. (2013)

ILIZASTIGUI MATOS, ANEYA. El tratamiento de problemas matemáticos interdisciplinarios en la formación del técnico medio en Agronomía. Editado en Revista electrónica Educación y Sociedad Revista Digital. Año 11 - Número 1. Enero-marzo 2013. Registrado ISSN 18119034 y RNPS/ 2073. (2013)

ILIZASTIGUI MATOS, ANEYA. “Temas de Didáctica de la Matemática”. Libro Electrónico. ISBN 978-959-18-0916-2. ( 2013)

MAÑALICH SUÁREZ Rosario “Interdisciplinariedad y didáctica”, en revista Educación. no. 94, mayo - agosto. (1998)

NÚÑEZ JOVER, JORGE. (2002): “Interdisciplinariedad: un reto para el PGI”, en Acercamientos a la interdisciplinariedad en la enseñanza-aprendizaje de las ciencias. IPLAC.

PLA LÓPEZ, R.V. y otros. La Pedagogía como ciencia desde un enfoque histórico cultural. 
Libro Electrónico. CEIP “Manuel Ascunce Domenech”. (2011).

TORRES SANTOMÉ, Jurjo (1994): Globalización e interdisciplinariedad: el currículo integrado. Editorial Morata. Madrid.

VIDAL ROJO C. (2007): Estrategia metodológica centrada en la determinación de nodos interdisciplinarios y la elaboración y aplicación de tareas integradoras. Tesis en opción al grado científico de Doctor en Ciencias Pedagógicas. Sancti Spíritus.

SILVESTRE ORAMAS, Margarita, ZILBERSTEIN TORUCHA, José (2002). Hacia una didáctica desarrolladora. Ed Pueblo y Educación. La Habana.

RECEBIDO EM 09 DE MARÇO DE 2014.

APROVADO EM 27 DE JULHO DE 2016. 